\title{
GENESIS AND BLACK HOLE UNIVERSE: THE THIRD DAY
}

\author{
Prof. PhD. Tianxi ZHANG, \\ Department of Physics, Chemistry, and Mathematics, \\ Alabama A \& M University, Huntsville, Alabama 35762, \\ UNITED STATES, \\ Email: tianxi.zhang@aamu.edu
}

\begin{abstract}
Recently the author has fully addressed the first two days of Genesis according to his well-developed black hole universe model (see Paper-I and Paper-II). In accordance with this new interpretation of Genesis, God first created the infinite entire universe called "earth" with matter named "water", and light, space and time, fundamental forces and motion. Then, he hierarchically structured the entire universe by separating the matter and space with infinite layers bounded by event horizons (called "vaults") and further formed our finite black hole universe. The efforts bridged the gap between Genesis and observations of the universe and brought us a scientific understanding of the Genesis. In this sequence study as Paper-III, we describe how God constructed the interiors of our finite black hole universe. It includes the formation of celestial objects by gathering the water or gravitationally collapsing the initial super fluidal matter under the sky or inside the even horizon of our black hole universe. These formed celestial objects could be stars and planets called dry grounds or lands, in which matter is not in the water state any more, and galaxies and clusters called, respectively, seas of stars and seas of galaxies.
\end{abstract}

Keywords: Genesis; Cosmology; Black Hole; Universe

\section{INTRODUCTION}

In Paper-I [1], the author has interpreted the first day of Genesis according to the black hole universe model, a new cosmology that the author recently developed [2-14]. The first day of creation was a long day. It contained the entire time period for God to create the three-dimensional (3D) infinite (or formless) and dark empty space (called earth in the book of Genesis), to make matter (named as water in the book of Genesis) and fill it into the empty space, to power the matter with motion and hence start the time, to create the fundamental forces among matter and issue matter with inertia of motion, and to generate light or radiation so that switched the entire space or the grand universe from darkness to brightness or from night to day (i.e. evening to morning)

In Paper-II [15], the author interpreted the second day of Genesis on the basis of the interpretation of the first day according to the black hole universe model. In the second day, God structured the entire space that he created in the first day into layers by separating the waters (i.e. the matter or super fluidal substance that God initially made and filled into the space) with vaults, which in physics can be understood as event horizons. God did this work by only setting the light speed as the speed limit for any matter and particles. From the entire infinite universe, which has infinite large radius and mass and infinitesimal density and temperature, to our finite black hole universe, which has finite mass, radius, density, and 
temperature, there are infinite layers, which are structured hierarchically and governed by the same fundamental laws of physics.

In the book of Genesis, the first through fifth sections of the first chapter describe the first day's work by God on creating the entire universe with matter and light, while the sixth through eighth sections of the first chapter describe the second day's work by God on structuring the entire universe with layers. Both the first and second days are not our earth day (i.e. 24 hours), the time needed for our earth to make one rotation about its axis. In the first two days, the Sun, planets including our earth, moon and even the interiors of our finite black hole universe were actually not formed and placed yet, and thus it is meaningless to say the earth day.

This paper as Paper-III attempts to self-consistently explain God's work on the third day in construction of the interiors of our finite black hole universe, including formation of stars and planets, galaxies and clusters, etc. With overall of this sequence of study, we aim at an attempt to develop author's well-developed new cosmological model, for revealing the mysteries of the universe and wiping out the discrepancy between science of cosmology and the book of Genesis. It provides a new interpretation of Genesis and meanwhile supports the black hole universe model in terms of Genesis. Through this effort, we will demonstrate the black hole universe model to be not only scientific because it reveals truths and selfconsistently explains observations of the universes, but also philosophical because it is complete and simply answers questions and overcomes difficulties without any non-testable hypothetical entities, and further theological because it is biblical and innovatively interprets the Genesis of the bible.

\section{GENESIS AND BLACK HOLE UNIVERSE: THE $3^{\text {rd }}$ DAY}

In this section, we interpret the third day of Genesis according to the black hole universe model. We again apply the New International Version (NIV) of the bible [16].

\subsection{Creating Celestial Objects in Our Universe}

${ }^{9}$ And God said, "Let the water under the sky be gathered to one place, and let dry ground appear." And it was so. ${ }^{10}$ God called the dry ground "land", and the gathered waters he called "seas". And God saw it was good. Here, the matter (i.e. the water or the God's initially created super fluidal matter) under the vault (i.e. inside the event horizon, e.g. our finite black hole universe) gathers (or gravitationally collapses) to one place appears (or produces) dry ground that God called it as "land". Since the God's created initial super fluidal matter is called the water as Paper-I described, then a dry ground or land is something without such water or not in the initial super fluidal state. Here, we suggest that the dry ground or land is an enough condensed, and thus not anymore super fluidal (i.e. gaseous, liquid, or solid) celestial object that God could stand firmly on, such as a star, a planet including our Earth, etc. The waters under the vault (i.e. inside the event horizon, e.g. our black hole universe) gather to many places so that appears or produce seas such as galaxies (i.e. the seas of stars including planets, etc.), clusters (i.e. the seas of galaxies), etc. Gathering water to one place appears a land or celestial body such as a star and planet, and gathering waters to many places appears many lands or celestial bodies (e.g. many stars and planets) that form a sea such as a galaxy. A galaxy is a sea of stars and a cluster is a sea of galaxies. The sea refers to a vast expanse or quantity of something.

In the Hebrew's era, concepts of galaxies and clusters were not built or developed, so that they were named as seas in the book of Genesis. In China, since the ancient time to the 
present, our galaxy, Milky Way, had being called as a river "Silver River" (or in Chinese "Yin He"). Figure 1 shows examples of seas of stars (i.e. galaxies) and seas of galaxies (i.e. clusters). This section of Genesis of the bible told us how God created stars, planets, galaxies, clusters, etc. from the initial super fluidal matter, i.e. the God's waters, through gravitational collapses under a vault or in a black hole universe (i.e. inside an event horizon). The matter or water flows downward across the vault and the vault rises upward, which explains the expansion of the black hole universe or spacetime. When a black hole accretes matter (or absorbs waters), it expands or enlarges its size. Observations indicate our black hole universe contains billions of galaxies and each galaxy contains billions of stars (Figure 2). For instance, our galaxy - the Milky Way - contains about hundred billions of stars. The Virgo cluster contains thousands of galaxies, in which the giant elliptical galaxy M87 is one of the largest and brightest galaxies. In general, galaxies formed from self-gravitationally collapsing of gas clouds are pancake-like [17, 18], as seen in the left panel of Figure 1.
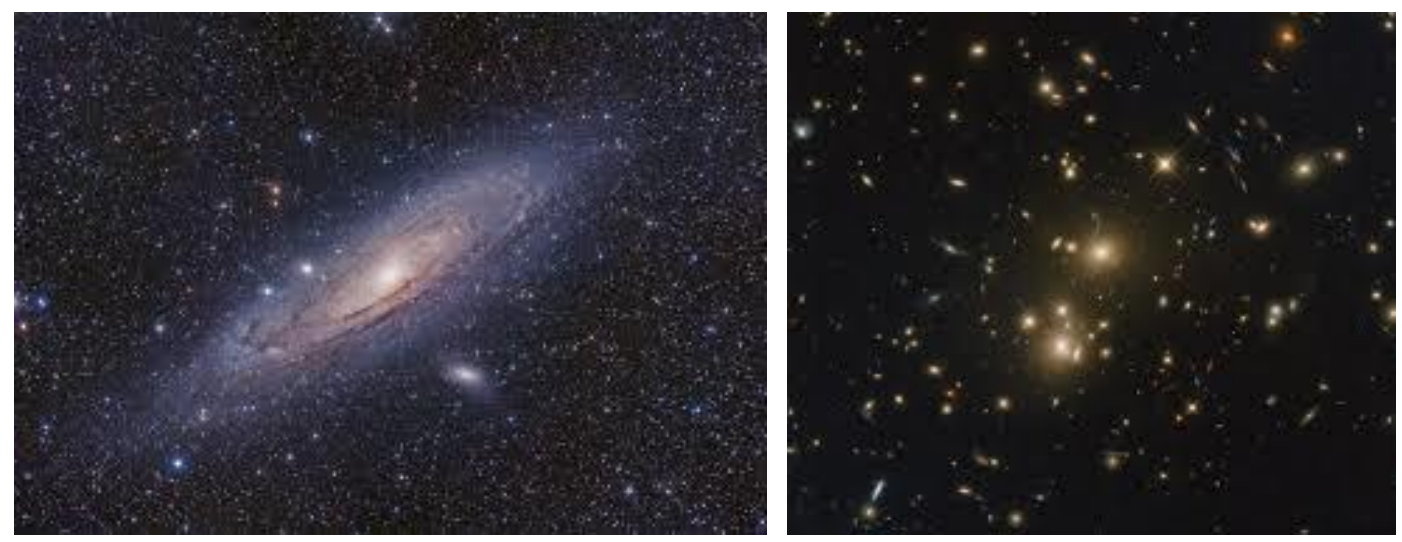

Fig. 1: A sea of stars - galaxy (left panel, credit by universetoday.com) and a sea of galaxies cluster (right panel, credit by galaxies: nasa.gov).

Once God created gravitation in the first day [1], matter gravitationally shrinking and collapsing are inevitable. Jeans instability causes the collapse of the fluidal matter or gas clouds and subsequent formation of celestial objects [19]. This occurs when the internal gas pressure or collision is not enough to prevent from gravitational collapse of a region filled with matter. Jeans length is the critical radius of a gas cloud where thermal energy is counteracted by gravity and Jeans mass is the mass of the gas cloud within Jeans length. They are determined, respectively, by

$$
L_{J} \sim \sqrt{\frac{k_{B} T}{G m}}, \quad \text { and } \quad M_{J} \sim L_{J}^{3} \sim \frac{k_{B} T}{G m} \sqrt{\frac{k_{B} T}{G m}},
$$

where $k_{B}$ is the Boltzmann constant, $G$ is the gravitational constant, $T$ is the gas temperature, $\rho$ is the gas mass density, and $m$ is the mass of gas particles (e.g. protons). For an instance, for a gas cloud with temperature of $10^{4} \mathrm{~K}$ and particle number density of $10^{14}$ protons $/ \mathrm{m}^{3}$, the Jeans length is about $10^{15} \mathrm{~m}$ and the Jeans mass is about $10^{32} \mathrm{~kg}$, the order in magnitude of the mass of a star. For other density and temperature, we may have a Jeans mass about the mass of a planet, a galaxy, or a cluster, etc. 


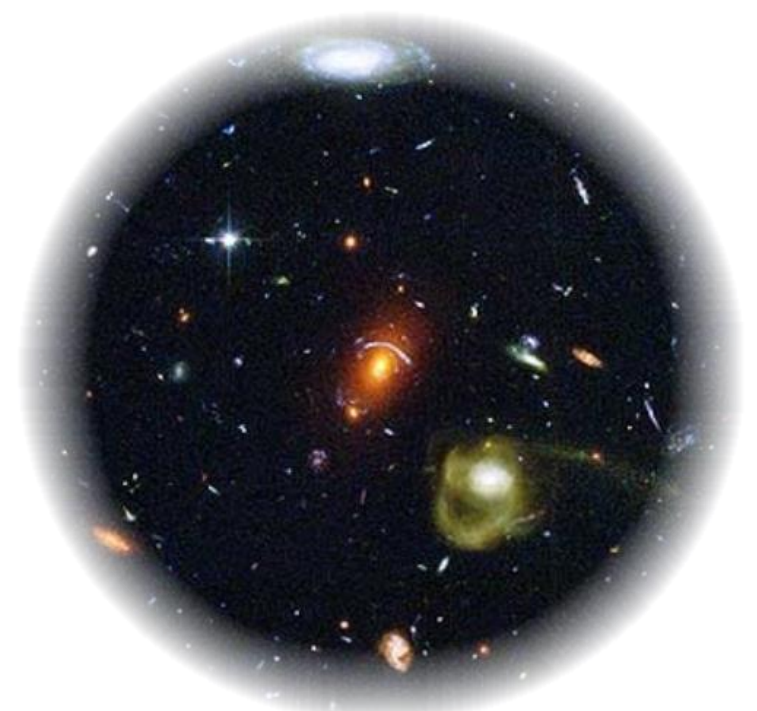

Fig. 2: Our universe contains billions of galaxies, each of which contains billions of stars (credit: kalilily.net). From the outside view, it is a black hole because nothing from the universe can escape.

\subsection{Initiating Nuclei Fusion and Nucleosynthesis for Star Formation}

At this moment of time, all astronomical or celestial objects formed are composed of mainly hydrogen atoms (may be ionized to be protons and electrons). They do not significantly shine or emit light because nuclear fusion could not occur due to that the Coulomb barrier between protons $\left(\sim 9.5 \times 10^{9} \mathrm{~K}\right.$ or $\left.820 \mathrm{keV}\right)$ is too much higher than the temperature of celestial bodies (e.g. for the Sun, $\sim 1.67 \times 10^{7} \mathrm{~K}$ or $1.5 \mathrm{keV}$ ). The book of Genesis called these types of highly condensed celestial objects as dry ground or land as described above. The stars due to no fusion occurrences do not emit visible light and thus darkness or in the evening or night. Figure 3 shows the potential energy as a function of the radial distance between two protons, given by [20]

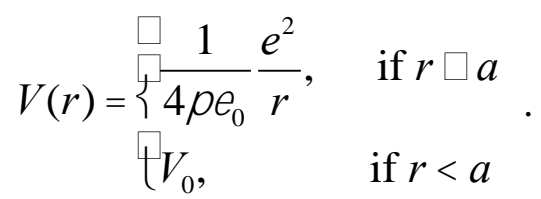

At $r=a \sim 1.78 \times 10^{-15} \mathrm{~m}$, about the proton's diameter, one can find the Coulomb barrier (or the maximum potential energy) to be $V(a)=U=820 \mathrm{keV}$ or 9.5 billions Kelvins. Here $V_{0}$ is the potential energy of the strong force with $-V_{0} \gg U$.

In accordance with the Maxwell-Boltzmann distribution function [21, 22], the number of protons with energy from $E$ to $E+d E$ in the object with temperature $T$ can be determined by

$$
\left.d N=\frac{2 N_{0}}{\left(k_{B} T\right)^{3 / 2}} \sqrt{E} \exp \square \frac{E}{k_{B} T}\right\rceil d E,
$$

where $N_{0}$ is the total number of protons. This distribution indicates that there is no single proton to be able to actually overcome the barrier in, for instance, a solar sized $\left(\sim 10^{30} \mathrm{~kg}\right)$ and hot $\left(\sim 10^{7} \mathrm{~K}\right)$ celestial body $[23,24]$. No fusion could be happened in these dry grounds or lands appeared or formed from the collapses of the matters or waters. Figure 4 plots that 
this reaction rate of protons in the Sun as a function of the core temperature [24]. It is seen that the reaction rate of protons is about zero (many orders in magnitude less than one proton per second), so that no nuclear fusion occurs in the core of the Sun if the core's temperature is equal to the conventional value $T_{\text {core }}=1.67 \times 10^{7} \mathrm{~K}$. For the reaction rate of protons to be the actually observed rate of $3.6 \times 10^{38}$ protons per second, the temperature of Sun's core must be about $1.3 \times 10^{8} \mathrm{~K}$ or above. Therefore, from the classical physics, the solar nuclear fusion is hardly to occur.

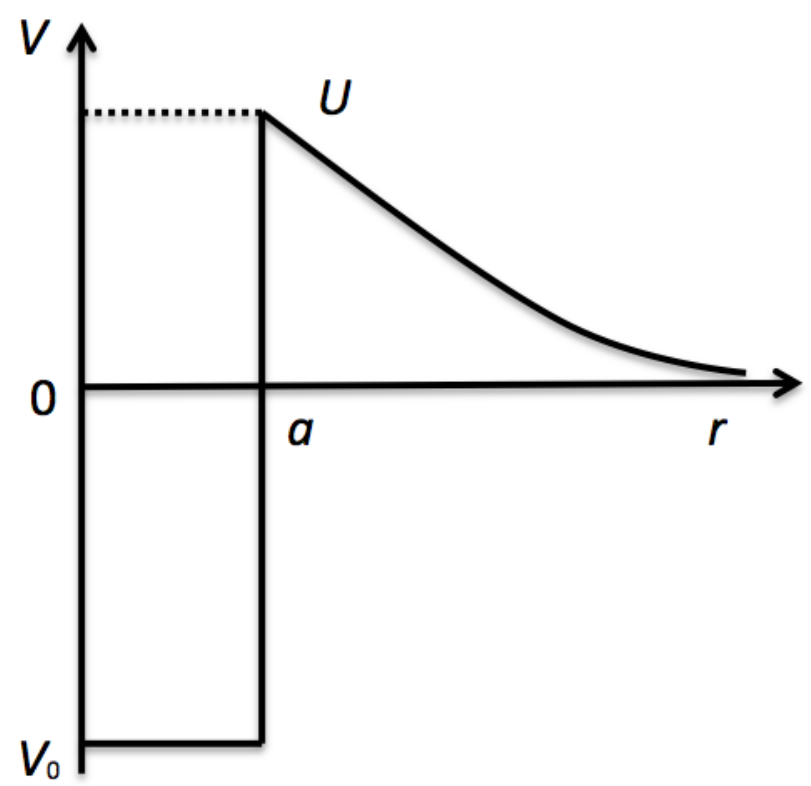

Fig. 3: The Coulomb barrier between two protons is given by $U=820 \mathrm{keV}$ or 9.5 billion Kelvins. The temperature of the core of a celestial body such as the Sun is only $1.5 \mathrm{keV}$ or 16.7 million Kelvins, about six hundred times lower than the barrier. In general a star size celestial object, before the fusion occurs, should have a lower temperature in comparison with a fusing one.

Then, to shine his created stars, God assigned particles of matter with also the wave property as the quantum physics has described. For a moving particle of mass $m$ and speed $v$, the de Broglie wavelength of the particle is given by [25]

$$
=\frac{h}{m v},
$$

where $h=6.62 \times 10^{-34}(\mathrm{~J} \mathrm{~s})$ is the Planck constant. The state of particle is described by a wave function $\Psi(\vec{r}, t)$, which is governed by the Schrodinger equation [26]

$$
i \hbar \frac{\partial \Psi(\vec{r}, t)}{\partial t}=-\frac{\hbar^{2}}{2 m} \nabla^{2} \Psi(\vec{r}, t)+V(\vec{r}) \Psi(\vec{r}, t),
$$

and statistically interpreted as the wave of matter or probability. The $|\Psi(\vec{r}, t)|^{2}$ is the density of probability of finding the particle at the time $t$ and the position $\vec{r}$. Meantime, light or radiation is set to have particle property and composed of photons, massless particles, whose energy is determined as [27, 28]

$$
E=h f,
$$


where $f$ is the light frequency. Both matter and massless radiation have duality of particle and wave.

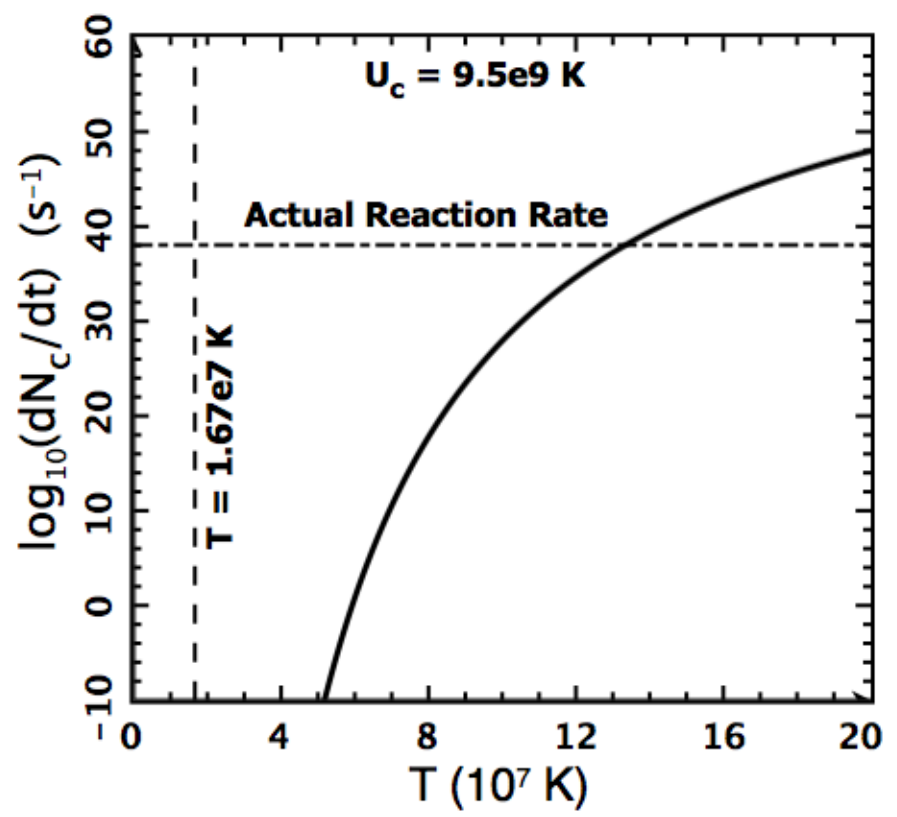

Fig. 4. The reaction rate of protons is plotted as a function of the Sun's core temperature in the case of without considering the quantum tunnelling effect [24]. The result indicates that no nuclear fusion can actually occur.

As particles behave like waves, quantum mechanics shows that particle waves can significantly tunnel through or penetrate the Coulomb barrier that is even though much higher than the energy of particle. This quantum tunnelling effect ignites or causes the start of nuclear fusion in the celestial bodies formed. According to the Gamow tunnelling probability [29]

$$
\left.P_{g}=\exp \sqrt{\frac{E_{g}}{E}}\right),
$$

one can determine the number of protons with energy from $E$ to $E+d E$ that can tunnel through or penetrate the Coulomb barrier as

$$
d N_{g}=P_{g} d N
$$

where $E_{g}$ is the Gamow energy given by

$$
E_{g}=m_{r} c^{2}()^{2}
$$

with $m_{p}$ the proton mass, $c$ the light speed in the vacuum, and $\alpha$ the fine-structure constant. This distribution (Eq. 8) exhibits a maximum called the Gamow peak that has energy to be hundred or more times less than the Coulomb barrier (Figure 5), so that the quantum tunneling effect greatly enhances the fusion reaction rate in the core of the astronomical objects and stars (e.g. the Sun). Taking the Sun as an example, we have that, without the quantum effect, the $10^{69}$ collisions among all (e.g. $10^{56}$ ) protons in one second in the core of the Sun with temperature about $1.5 \mathrm{keV}$ cannot have a single collision of overcoming the 
Coulomb barrier. With the quantum effect, the percentage of tunneling through the Coulomb barrier raises to the order about one per million collisions (i.e. $10^{-6}$ ). In other words, the $10^{63}$ collisions among the $10^{56}$ protons in one second in the core of the Sun may penetrate the Coulomb barrier and form diprotons. The rate of formation of deuterons from diprotons via the $\beta^{+}$-decay must be about $10^{-25} \mathrm{~s}^{-1}$ in order for the fusion rate of diprotons to deuterons in the core of the Sun to be the order of $3.6 \times 10^{38} \mathrm{~s}^{-1}$, so that the Sun can shine at the observed luminosity about $3.85 \times 10^{26} \mathrm{~W}$.

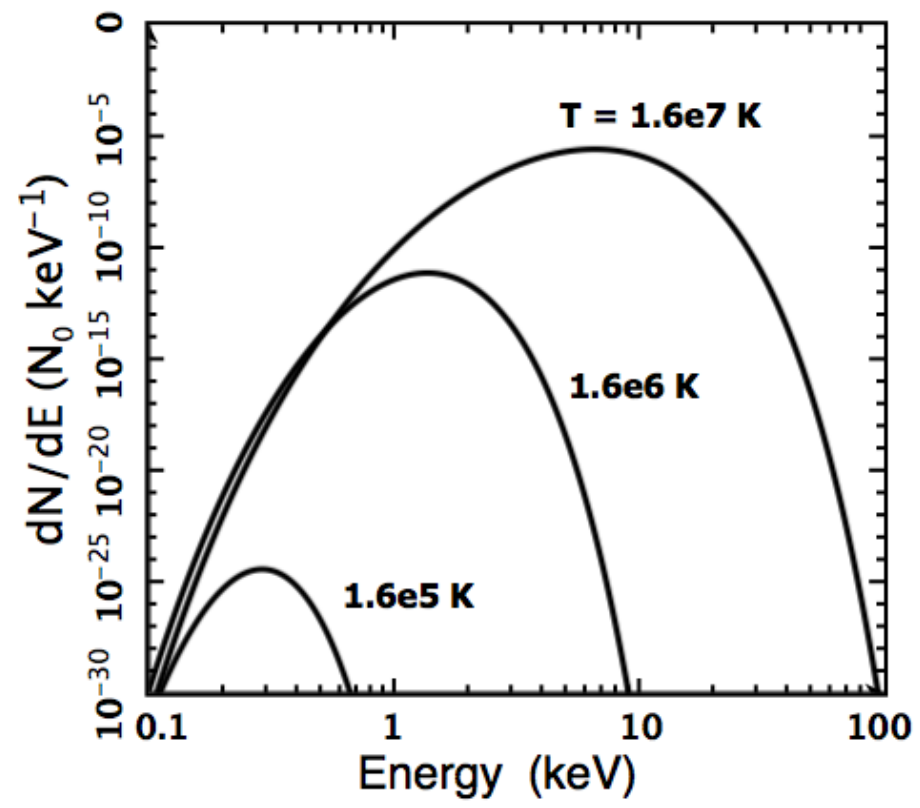

Fig. 5: Gamow peaks. The number of tunneling protons per unit energy in the core of astronomical object is plotted as a function of the proton energy in three cases of the core temperature.

However, as pointed out recently by the author [24], in the conventional analysis and calculation with the Fermi theory of the $\beta^{+}$-decay, the significant wave function of twoscattering protons was usually used for the inefficient wave function of the diproton outside the potential energy well [30]. This is not physical and greatly weakens the wave function of the diproton inside the potential energy well, so that leads to the probability for a diproton to form a deuteron via a $\beta^{+}$-decay to be extremely under calculated [24]. In other words, the rareness of $\beta^{+}$-decay in diprotons may not be rare enough to inhibit the solar nuclear fusion or lower the fusion rate by an order of magnitude 25, in order to stop the Sun's instantaneous explosion and have the currently observed luminosity. Quantum tunneling effect allows many diprotons formed in the Sun's core, but the probability for a diproton to form a deuteron via a $\beta^{+}$-decay may not be lower than that for a diproton to separate back to two protons by an order of magnitude 25 . Observations have only given an upper bound that a diproton (or helium- 2 nucleus) gets $\beta^{+}$-decay by less than one per ten thousands, i.e. $<0.01 \%$ [31], which is many orders higher in magnitude than $10^{-25}$.

Recently, the author of this paper has proposed a new mechanism of inhibition that can significantly reduce the fusion reaction rate and thus effectively prevent the Sun from an instantaneous explosion. It has been suggested that the core of the Sun involves a significant physical effect or inhibitor called plasma oscillation or wave, which significantly reduces the 
electric permittivity of the core plasma. A significantly reduced electric permittivity will greatly raise the Coulomb barrier as well as efficiently lower the Gamow tunneling probability. These changes lead to the Gamow peak greatly shift to the region of higher energies of particles. Quantitative study by the author has indicated that, if the frequency $\omega$ of the plasma oscillations or waves that are globally generated in the core plasma of turbulences (e.g. Longmuir waves) is about 1.28 times the plasma frequency $\omega_{p}$, the Sun can have the actual fusion rate or shine on at the currently observed luminosity, as shown in Figure 6. Therefore, in addition to the quantum tunneling effect, the plasma oscillations may play also an essential role in the Sun's nuclear fusion and power emission. The quantum tunneling effect makes the fusion to occur, while the plasma oscillations in association with the weak $\beta^{+}$-decay of diprotons guarantees the Sun to be not exploded. The author also suggested that a supernova explosion occurs when plasma oscillations in the core of a star at the end of its life are significantly weakened in intensity or changed in frequency that cause the heavy ion fusion to be significantly speeded up and the huge amount of energies and neutrinos to be instantaneously emitted. The result of this study also gives important implications to plasma nuclear fusion in laboratory and solar neutrino missing problem.

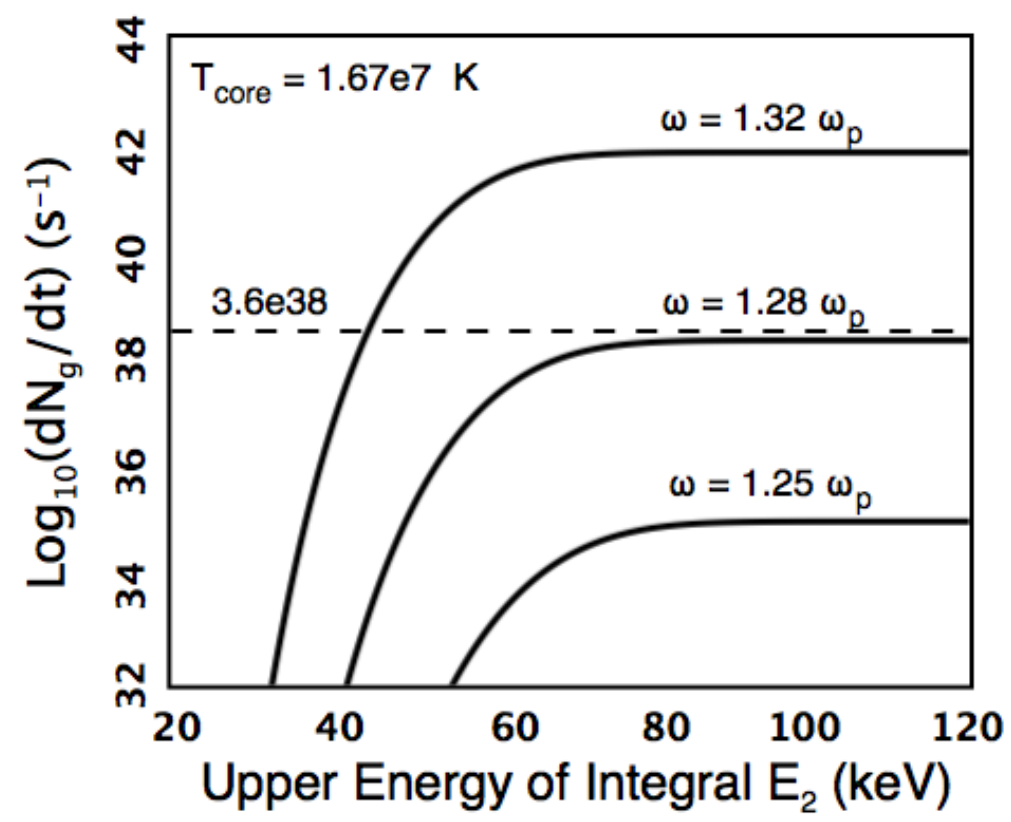

Fig. 6. The reaction rate of protons in the core of the Sun with the plasma oscillation effect [24]. The number of adequate collisions per second between protons is plotted as a function of the upper energy of the integration.

At the end of its life, a star runs out proton-proton fusion that varies the plasma composition and oscillations, so that cause the proposed efficient inhibition of plasma fusion reaction to be ineffective. When plasma oscillations in the core of a star at the end of its life are significantly weakened in intensity or changed in frequency, fusion reactions among heavy nuclei will be significantly speeded up and out of control that leads to huge amount of energies and neutrinos to be instantaneously emitted. This provides us another alternative mechanism for supernova explosions, in addition to the previously proposed and welldeveloped models of supernova explosions driven by magnetohydrodynamic (MHD) rotation 
[32], acoustic waves [33], neutrinos [34], and gravitational field shielding [35]. After supernova explosion, the core of a star forms a black hole if the star's initial mass is over 20 solar masses, otherwise forms a neutron star. A star with mass less than 8 solar masses usually ends as a white dwarf after most of its outer material is expelled. The existence of black hole in the nature was recently conformed by LIGO, which first ever detected gravitational waves from mergers of binary black holes [36]. The observational discovery of neutron stars was done in the middle of the 1960s from the measurement of pulse-like radio emissions from the Crab Nebula [37,38]. The conventional interpretation to the observed pulse-like radio emissions was based on the lighthouse model of pulsars as fast rotating neutron stars [39]. Recently, the author developed a physical model of pulsars as gravitational field shielding and oscillating neutron stars [40] and quantitatively interpreted the emission characteristics of pulsars, in accordance with the author well-developed 5D fully covariant theory of gravitation [41] and the physics of radiation from thermal and accelerating charged particles [42], as shown in Figure 7.

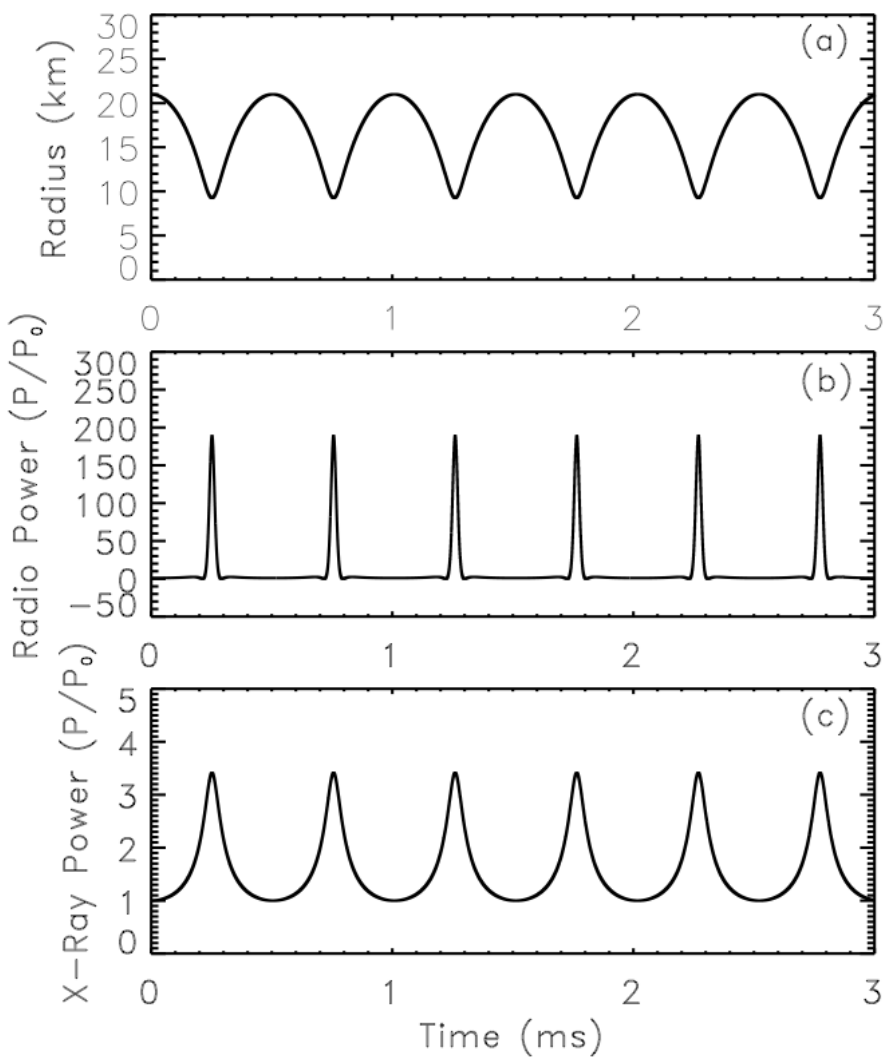

Fig. 7. Oscillation of a neutron star with 1.5 solar masses versus synchronous emissions of the Dirac Delta shape radio pulses and the Lorentzian shape X-ray oscillations [40]. The radial distance (a), the power of radio emission (b), and the power of X-ray emission (c) are plotted as functions of time.

At the center of a galaxy, there in general exists a massive black hole. The Sagittarius (Sgr) A* is believed to be a massive black hole with 4.3 million solar masses at the center of the Milky Way [43,44]. It is usually quite and faint, but occasionally emit intensive flares of X-rays. Recently, NASA Chandra X-ray Observatory and other missions such as Swift, 
NuStar, XMM-Newton, and Roast have discovered intensive and rapid X-ray flares, at a rate of about once a day from Sgr A*, with luminosity at the peak up to a few times $10^{35} \mathrm{erg} / \mathrm{s}$ $[45,46]$. To explain the mysterious X-ray flares, astronomers have suggested that there exists a gas cloud around Sgr A* containing hundred-trillions of asteroids, comets, and planets that are stripped from their parent stars by the tidal forces of the massive black hole. When these objects rain down or are accreted onto the massive black hole, X-ray flares take place via physical processes such as the non-thermal synchrotron emission [47], the inverse-Compton scattering [48], and stochastic electron acceleration [49]. To emit the high-energy X-rays detected, an object that was striped from its parent star had to be torn apart into gases during its falling and the gases when arriving nearly at the massive black hole had to spike to hundreds of million degrees Celsius, which is ten or more times hotter than the center of the Sun. However, why the gases heat up so suddenly and efficiently on a regular basis is still poorly understood. Especially, Sgr A* may not be able to gravitationally tear an asteroid into parts as small as a human body, because the gravitational field difference between the head and feet of a 2-meter height person, who stands on Sgr A* surface is only $10^{-3} \mathrm{~m} / \mathrm{s}^{2}$. Up to the date, astrophysicists are still out on what really caused these giant X-ray flares from Sgr A*. The mechanism for the origin of X-ray flares from the galactic center is still a mystery and in pending for a physical explanation. Recently, the author developed a new mechanism for Xray flares from $\mathrm{Sgr} \mathrm{A}^{*}$ as emissions of dynamic massive black hole at the Milky Ways center, in accordance with the author well-developed black hole model of the universe [12]. An any sized black hole, when accreting matter, becomes dynamic and breaks its event horizon, which leads to the inside hot (or high-frequency) blackbody radiation leaking out of it and produces an X-ray flare or burst. The energies and spectra of X-rays calculated can explain the current measurements of X-ray flares from Sgr A* including its steady emissions (Figure 8). It also predicts big events that possibly occurred in the past or will possibly occur in future at our galactic center, and compare the predicted intensive events with the measurements of strong X-ray flares from other normal and active galactic centers.

Quasars are quasi-stellar objects, from which light is extremely shifted toward the red [50]. Quasars are generally believed to be extremely luminous galactic centers powered by supermassive black holes with masses up to billions of solar masses [51]. It is usually suggested that the material (e.g., gas and dust) falling into a supermassive black hole is subjected to enormous pressure and thus heated up to millions of degrees, where a huge amount of thermal radiation including waves, light, and X-rays give off [52]. However, the density of the falling material, if it is less dense than the supermassive black hole, is only about that of water. In other words, the pressure of the falling gas and dust may not go such high required for a quasar to emit energy as amount of that emitted by hundred billions of the Sun. The author proposed a possible explanation for quasars to ignite and release a huge amount of energy in accord with the black hole universe model [5]. General relativity tells us that a main sequence star will, in terms of its mass, form a dwarf, a neutron star, or a black hole. After many stars in a normal galaxy have run out of their fuels and formed dwarfs, neutron stars, and black holes, the gravity cause the galaxy to eventually collapse and form a supermassive black hole with billions of solar masses. It has been shown that this collapse can lead to the extremely hot stellar black holes to merge each other and further into the massive black hole at the center and release intense thermal radiation energy as great as a quasar emits. When the stellar black holes of a galaxy collapse and merge into a supermassive black hole, the galaxy is activated and a quasar is born (Figure 9). The observed distant quasars were donuts from the mother universe. They were actually formed 
in the mother universe as little sisters of our universe. After the quasars entered our universe, they became our universe's child universes. The results from this quasar model are consistent with observations. In addition, the author developed an new redshift mechanism called electric redshift in terms of his 5D fully covariant theory of gravitation to alternatively explain the non-cosmological components of quasar large redshifts [53].

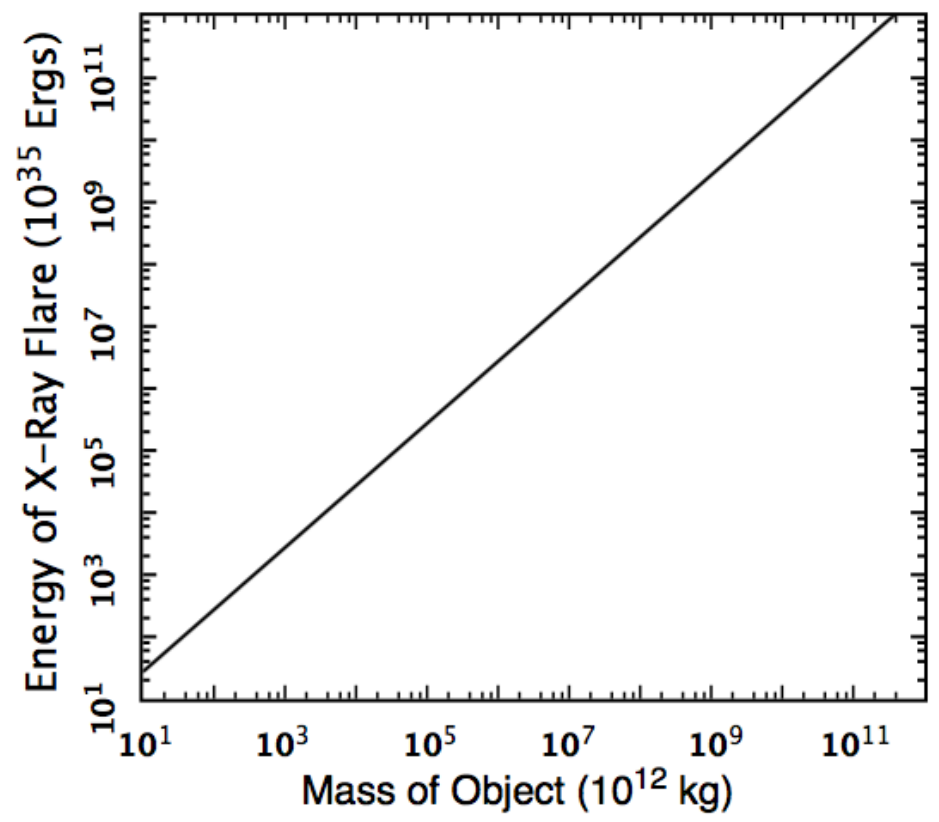

Fig. 8: The energy of X-ray flares from Sgr $A^{*}$ versus the mass of the object accreted [12].

\subsection{Creating Plants and Trees on Our Earth}

${ }^{11}$ Then God said, "Let the land produce vegetation: seed-bearing plants and trees on the land that bear fruit with seed in it, according to their various kinds. "And it was so. ${ }^{12}$ The land produced vegetation: plants bearing seed according to their kinds and trees bearing fruit with seed in it according to their kinds. And God saw that it was good. ${ }^{13}$ And there was evening, and there was morning - the third day. Here God chose one land (i.e. celestial body or planet) that he created as our land or our Earth to produce plants and trees that bear fruits with seeds according to their various kinds. God let our Earth produce or grow vegetation, including plants that bear seeds according to their kinds and trees that bear fruits with seeds in them according to their kinds. Since this part does not belong to physical science, the author cannot appropriately describe the details. Therefore, the third day was the day of structuring the interiors of our finite black hold universe by creating celestial objects from the gravitationally collapsing matter such as stars including planets and our Earth, on which plants and vegetation were produced. To shine the stars, God assigned matter and light or massless radiation with duality of particle and wave. This leads to fusion reaction to occur in the core of stars and power emissions from the stars. It was the time for God to form stars (lightness or day) including galaxies (seas of stars) and clusters (seas of galaxies) from the initial waters (darkness or night) and to create our planet, the Earth, that grows plants and vegetation. 


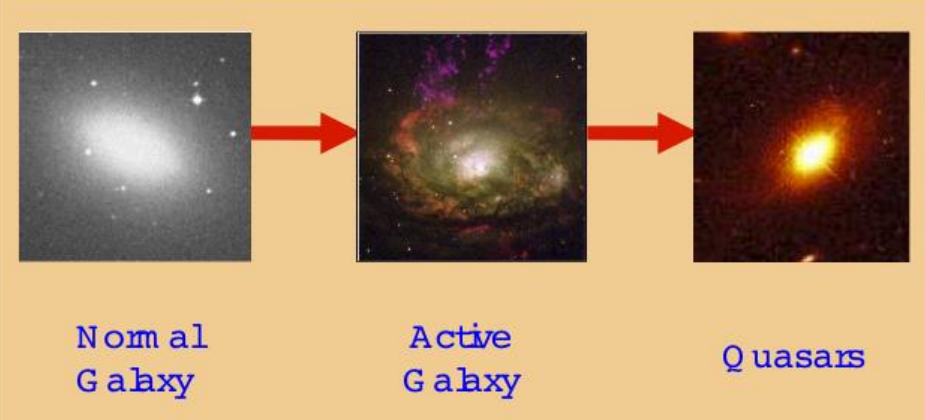

Fig. 9: Formation of quasars [5]. A normal galaxy evolves into an active one and ends by a quasar (Credit: Images of Hubble Space Telescope).

\section{CONCLUSION}

We have interpreted the third day of Genesis as the day of forming stars including planets, galaxies, and clusters in our finite black hole universes. In the third day, God constructed the interiors of our finite black hole universe. The work includes the formation of celestial objects by gathering the waters or gravitationally collapsing the initial super fluidal matter under the sky or inside the even horizon of our black hole universe. These formed celestial objects could be stars and planets called dry grounds or lands, in which matter is not in the water state any more, and galaxies and clusters called, respectively, seas of stars and seas of galaxies. Stars luminously shine when fusion occurs after particles of matter were assigned with the wave property. God further selected one land (i.e. our Earth) for plants to grow and further for humans to live.

\section{BIBLIOGRAPHY:}

[1] Zhang, T. X., "Genesis and Black Hole Universe I: The First Day", International Journal of Theology, Philosophy, and Science, 6 (2020), 54-67

[2] Zhang, T. X., "A New Cosmological Model: Black Hole Universe", American Astronomical Society $211^{\text {th }}$ Meeting, Abstract \#152.04 (2007)

[3] Zhang, T. X., "A New Cosmological Model: Black Hole Universe", Progress in Physics, 5 (2009), 311

[4] Zhang, T. X., "Cosmic Microwave Background Radiation of Black Hole Universe", Astrophysics and Space Science, 330 (2010), 157-165

[5] Zhang, T. X., "Quasar Formation and Energy Emission in Black Hole Universe", Progress in Physics, 3 (2012), 48-53

[6] Zhang, T. X., "Key to the Mystery of Dark Energy: Corrected Relationship Between Luminosity Distance and Redshift", Progress in Physics, 9 (2013), 33-38

[7] Zhang, T. X. "A New Mechanism for Gamma Ray Bursts: Emissions of Dynamic Black Holes", Proceeding of Gamma Ray Burst 2013 Symposium, SNSN-323-63 (2013)

[8] Zhang, T. X., and Frederick, C. "Acceleration of Black Hole Universe", Astrophysics and Space Science, 349 (2014), 567-573

[9] Zhang, T. X., "Gamma Ray Bursts of Black Hole Universe", Astrophysics and Space Science, 358 (2015), article id. \#14, 8 pp

[10] Zhang, T. X., Nika P., and Guggilla P., "Energy and Spectra of Gamma Ray Bursts from Mergers of Binary Black Holes", Gamma-Ray Burst Symposium, article. Id. 4028.pdf (2016).

[11] Zhang, T. X., "Principle of Spacetime Black Hole Equivalence", Progress in Physics, 12 (2016), 353-361

[12] Zhang, T. X., Wilson, C., and Schamschula, M. P., "X-Ray Flares Sagittarius A* and Black Hole Universe", Progress in Physics, 12 (2016), 61-67

[13] Zhang, T. X., "Mach's Principle to Hubble's Law and Light Relativity", Journal of Modern 
Physics, 9 (2018), 433-442

[14] Zhang, T. X., "The Principles and Laws of Black Hole Universe", Journal of Modern Physics, 9 (2018), 1838-1865

[15] Zhang, T. X., "Genesis and Black Hole Universe I: The Second Day", International Journal of Theology, Philosophy, and Science, 7 (2020), 5-19

[16] New International Version of the Bible, www.biblehub.com/niv /genesis/1.htm.

[17] Li, X. Q., "Nonlinear Structures of Self-Gravitating Systems", Astronomy and Astrophysics, 227 (1990), 317-323

[18] Zhang, T. X., and Li, X. Q., "Nonlinear Structures of Self-Gravitating Systems in Stable Modes", Astronomy and Astrophysics, 294 (1995), 339-344

[19] Jean, J. H., "The Stability of a Spherical Nebula", Philosophical Transactions of the Society A., 199 (1902), 312-320

[20] Coulomb, P. M., "Histoire de Academie Royale des Sciences", (1875), 569-577

[21] Maxwell, J. C., "On the Motions and Collisions of Perfectly Elastic Spheres", Philosophical Magazine and Journal of Science, 19 (1860), 19-32

[22] Boltzmann, L., "Weitere Studien uber das Warmegleichgewicht unter Gasmolekulen", Sitzungsber. Kais. Akad. Wiss., 66 (1872), 275-370

[23] Zhang, T. X. and Ye, M. Y., "Nuclear Fusion with Coulomb Barrier Lowered by Scalar Field", Progress in Physics, 15 (2019), 191-196

[24] Zhang, T. X., "The Role of Plasma Oscillation Played in Solar Nuclear Fusion", Progress in Physics, 17 (2021), 93-98

[25] de Broglie, L., "Recherches sur la Theorie des Quanta", Annales de Physiques, 10 (1925), 22128

[26] Schrodinger, E., "An Undulatory Theory of the Mechanics of Atoms and Molecules", Physical Review, 28 (1926), 1049-1070

[27] Planck, M., "On the Theory of the Energy Distribution Law of the Normal Spectrum", Verhandl. Dtsch.Phys. Ges., 2 (1900), 237-245

[28] Einstein, A., "On a Heuristic Point of View Concerning the Production and Transformation of Light", Annalen der Physik, 17 (1905), 132-148

[29] Gamow, G., "Zur Quantentheorie des Atomkernes", Z. Physik, 51 (1928), 204-212

[30] Bethe, H. T., "The Formation of Deuterons by Proton Combination", Physical Review, 54 (1938), 248-254

[31] en.wikipedia.org/wiki/Isotopes \ of \ helium

[32] Meier, D. L., Epstein, R. I., Arnett, D., and Schramm, D. N., "Magnetohydrodynamic Phenomena in Collapsing Stellar Cores", Astrophysical Journal, 204 (1976), 869-878.

[33] Burrows, A., Livne, E., Dessart, L., Ott, C. D., and Murphy, J., "Features of the Acoustic Mechanism of Core-Collapse Supernova Explosions". Astrophysical Journal, 655 (2007), 416-433.

[34] Colgate, S. A., and White, R. H., "The Hydrodynamic Behavior of Supernovae", Astrophysical Journal, 143 (1996), 626-681

[35] Zhang, T. X., "Gravitational Field Shielding and Supernova Explosions". Astrophysical Journal Letters, 725 (2010), L117-L121

[36] Abbott, B. P. et al., "Observation of Gravitational Waves from a Binary Black Hole Merger", Physical Review Letters, 116 (2016), id. 061102

[37] Hewish, A., and Okoye, S. E., "Evidence for an Unusual Source of High Radio Brightness Temperature in the Crab Nebula", Nature, 207 (1965), 59- 60

[38] Hewish, A., Bell, S. J., Pilkington, J. D. H., Scott, P. F., and Collings R. A., "Observation of a Rapidly Pulsating Radio Source", Nature, 217 (1968), 709-713

[39] Lyne, A. G., and Graham-Smith, F., Pulsar Astronomy, Cambridge Univ. Press, Cambridge, 2012

[40] Zhang, T. X., "A Physical Model of Pulsars as Gravitational Field Shielding and Oscillating Neutron Stars", Progress in Physics, 11 (2015), 110-116

[41] Zhang, T. X., "The 5D Fully-Covariant Theory of Gravitation and Its Astrophysical Application", Galaxies, 3 (2015), 18-51

[42] Jackson, J. D., Classical Electrodynamics, 3rd Edition. John Wiley and Sons, (1999), p. 665

[43] Balick, B., and Brown, R. L., "Intense Sub-arcsecond Structure in the Galactic Center". Astrophysics and Space Science, 194 (1974), 265-270 
[44] Schodel, R. et al., "A Star in a 15.2-Year Orbit Around the Supermassive Black Hole at the Centre of the Milky Way", Nature, 419 (2002), 694- 696

[45] Porquet, D. et al., "XMM-Newton Observation of the Brightest X-ray Flare Detected so far from Sgr A*", Astronomy and Astrophysics, 407 (2003), L17-L20

[46] Neilsen, J. et al., "A Chandra/HETGS Census of X-ray Variability from Sgr A* during 2012", Astrophysics Journal, 774 (2013), article id. 42

[47] Kusunose, M., and Takahara, F., "Synchrotron Blob Model of Infrared and X-ray Flares from Sagittarius A*”, Astrophysical Journal, 726 (2011), article id. 54, 6 pages

[48] Yusef-Zadeh, F. et al., "An Inverse Compton Scattering Origin of X-ray Flares from Sgr A*", Astronomical Journal, 144 (2012), article id. 1, 10 pages

[49] Liu, S. M., Fulvio, M., and Yahe, F., "Stochastic Electron Acceleration during the NearInfrared and X-ray Flares in Sagittarius A*”. Astrophysical Journal, 636 (2006), 798-803

[50] Burbidge, E. M., "Quasi-Stellar Objects", Annual Review of Astronomy and Astrophysics, 5 (1967), 399-452

[51] Rees, M. J., "Black Hole Models for Active Galactic Nuclei", Annual Review of Astronomy and Astrophysics, 22 (1984), 471-506

[52] Wyithe, J., and Stuart, B., "Loeb A. Self-regulated Growth of Supermassive Black Holes in Galaxies as the Origin of the Optical and X-ray Luminosity Functions of Quasars", The Astrophysical Journal, 595 (2003), 614-623

[53] Zhang, T. X., Electric Reshift and Quasars, Astrophysical Letters, 636 (2006), L61-L64 